\title{
Extension Association Decision Method of Feature Processing Scheme Based on Feature Recognition
}

\author{
Jinmei $\mathrm{Gu}^{\mathrm{a}}$, Fengli Huang ${ }^{\mathrm{b}}$ \\ School of Mechanical and Electrical Engineering, Jiaxing University, Zhejiang, 314033, China \\ a jmgu8037@163.com, b zjxuhfl301@163.com
}

Keywords: feature machining, feature recognition, extension relationship, process scheme

\begin{abstract}
At present, the feature machining is recognized automatically for the typical CAM software, but there are some shortcomings in the technological rule base which support feature machining scheme. First, the classification and data model and the identification method of manufacturing feature are given. Then, the mapping of parts feature model to the processing schemes is given; the main variables are defined as follows: blank material, size tolerance, surface roughness and shape characteristics. The processing scheme of the non-hole class characteristics and hole class characteristics are defined according to the difference of shape feature. Based on above, association method of the recognition feature machining scheme is proposed based on extension comprehensive correlation functions. At last, the application instance shows the better operability of this method, and the automatic generation of feature machining scheme has come true.
\end{abstract}

\section{Introduction}

With the rapid development of 3D CAD software, entity modeling building technology has grown pretty mature, much commercial modeling software with superior performance have emerged, the system application of CAPP/CAM/CAE associated with the physical model is becoming more and more popular [1]. Three-dimensional entity model is complete and accurate definition of the three-dimensional entity's geometry information and topology information, and many engineering application problems have been solved successfully. According to the traditional working way of CAD/CAM system, CAM system obtain the points, lines, faces or entities of the product geometric data model for the driver object directly or indirectly (through neutral file), the tool path is generated, the NC code is gotten in the form of a cutting tool positioning through post processing, which is transferred to the CNC machine tool to finish the machining[2]. The problem is how to carries on NC program directly for solid model to improve programming efficiency.

The feature processing method has been preliminarily used in the existing mature CAM system software. The FBM Mill of MasterCAM can detect the all processing characteristics of parts within the scope of plane through the analysis of parts waiting to be processed, and the selected features are required to complete processing, the all 2D milling cutting tool path are generated automatically. CAMWorks is CAM software integrated in SolidWorks completely, which realize the seamless linking with SolidWorks, AFR (Automatic Feature Recognition) and IFR(Interactive Feature Recognition) can be done for the processing characteristics of SolidWorks design model. The manufacturing Feature can be built directly in the parts model in the feature machining unit of UG $\mathrm{NX}$, realize the geometry information recognition and extraction in many ways. Tell the system how to identify characteristics through processing template set, and assign processing method for the characteristics. Thus, the geometrical characteristics of the solid model as processing, programming method on the basis of characteristic parameters can reduce the input of processing object and parameters, and model features as the link, connect the CAD/CAM system, realize the data dynamic linking. The corresponding case database and knowledge base need to be set up in the characteristics processing module in order to form process parameters of model, built processing procedure structure tree, realize the automatical generation of NC machining program. In this paper, extension 
comprehensive correlation method was used to the matching retrieval of manufacture feature, to automatically realize dynamic relation between CAD/CAM data.

\section{Data model and classification of manufacture characteristics}

Reference to the definition of the data model about the parts processing part in ISO14649, the processing data model includes two parts, work piece and processing scheme. Work piece is composed of a series of processing characteristics, the processing scheme is the sequence of processing steps, processing work step consists of machining features and operation ${ }^{[3,4]}$. This structured data model can fully describe all work piece geometry information and information of processing and manufacturing, which is very suitable for information description of the processing characteristics.

Manufacture feature specifically refers to the shape of materials removed from blank parts in order to get the final shape of parts. The manufacturing characteristics of parts include the processing features, excessive characteristics and repeating characteristics. Processing features include multiple spindle, composite features, thread, printing, etc., multi-axis characteristics include hole, slot, steps, plane, convex platform, outline, etc. The relationship between them is shown in figure 1.

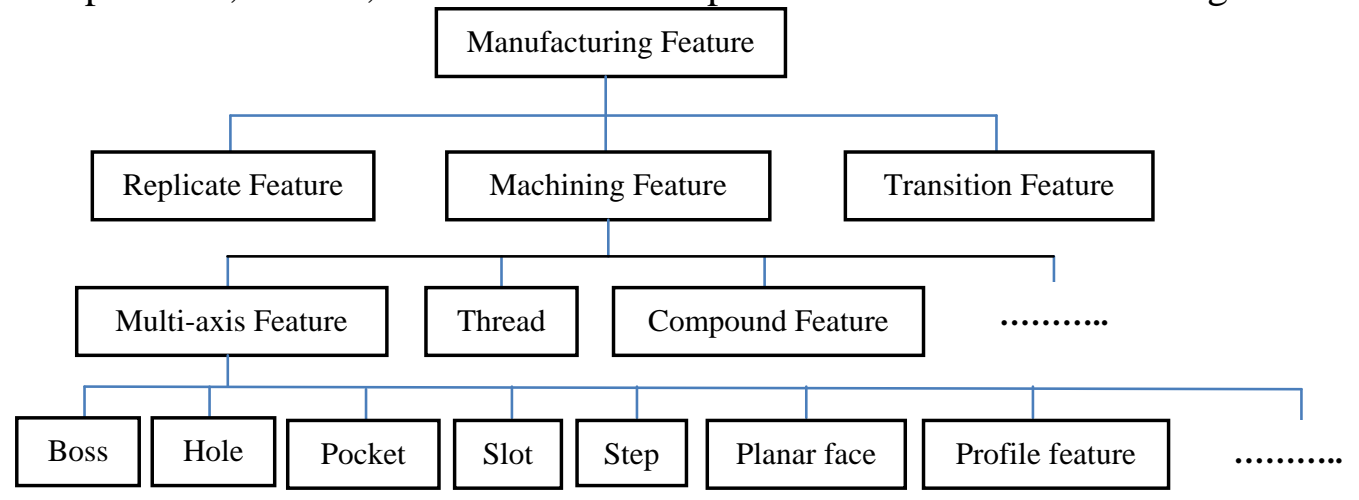

Fig.1 type and relationship of manufacturing characteristic

\section{Manufacture feature recognition and feature processing rules}

The identification method of manufacture feature.Feature recognition is to find out the set of correlation surface from the surface of parts, each collection corresponds to a feature. The characteristics of the high level such as groove and hole can be recognized from the points, lines and planes of the low level characteristics of parts. Feature recognition method on the whole can be divided into three categories: feature recognition method based on boundary matching, feature recognition method based on three-dimensional decomposition and feature recognition method based on the machining surface - resource mapping [5].

The existing mature three-dimensional CAD software has the function of automatic identification. Open the existing component model or build new parts model in SolidWorks, click on the button of feature, CAMWorks will recognize automatically all the processing features, read all the information contained, and arrange in order on the feature tree according to the order of feature recognition.

The processing rules of feature machining.The processing scheme of feature machining is closely related to the parts material and process properties and shape characteristics, technological properties are as follows: size tolerance, geometrical tolerance and surface roughness. Geometrical tolerance is related to machine tool accuracy, the clamping precision in addition to the processing scheme. But overall, in the certain case of various processing conditions, there is a proportional relationship between shape tolerance, size tolerance and the surface roughness. The main factor to be considered is blank material, size tolerance, surface roughness and feature shape.

(1) Considering the needs of the relational mapping model, Blank material is divided into casting material, non-ferrous metals, refractory material and hardening materials according to the difference 
of mechanical property such as hardness of commonly used processing materials, which is regarded as .

(2) The size tolerance level is regarded as IT, the achieved tolerance grade of the selecting machining method is divided into economic size tolerance and feasible size tolerance, its scope is $I T_{L}$ - $I T_{H}$ and $I T_{L}^{\prime}-I T_{H}^{\prime}$ respectively.

(3) The surface roughness values are expressed in Ra, the surface roughness that processing method can reached is divided into economic surface roughness and feasible surface roughness, its scope is $R a_{L}-R a_{H}$ and $R a_{L}^{\prime}-R a_{H}^{\prime}$.

(4) Feature shape are divided into curved surface class characteristics and plane class characteristics according to different final forming surface, also, it is divided into the mold cavity and surface processing according to the knife road layers which is expressed as $F=\left[F_{1}, F_{2}, F_{3}, F_{4}\right]$ $=[$ surface cavity, the plane cavity, surface, plane]. The biggest tool size of the characteristics are defined as $D_{\mathrm{M}}$, the tool size to complete all processing is defined as $D_{\mathrm{L}}$, obviously, $D_{\mathrm{L}} \leq D_{\mathrm{M}}$, The characteristics of inner cylinder surface (such as hole) are defined as holes class processing characteristics, which is expressed as $F=\left[F_{1}\right]=$ [hole].

The corresponding processing scheme of non-hole class characteristics is called $X$ processing scheme, as shown in table 1 , which is expressed as $X=\left[X_{1}, X_{2}, X_{3}, X_{4}\right]$. The corresponding processing scheme of hole class characteristics is called $Z$ processing scheme, as shown in table 2 , which is expressed as $Z=\left[Z_{1}, Z_{2}, Z_{3}, Z_{4}, Z_{5}, Z_{6}, Z_{7}, Z_{8}, Z_{9}\right]$.

Table $1 X$ processing scheme

\begin{tabular}{clcccc}
\hline Code & $\begin{array}{l}\text { Processing } \\
\text { method }\end{array}$ & $\begin{array}{l}\text { Economic } \\
\text { size tolerance }\end{array}$ & $\begin{array}{c}\text { Feasible size } \\
\text { tolerance }\end{array}$ & $\begin{array}{c}\text { Economic } \\
\text { roughness }\end{array}$ & $\begin{array}{c}\text { Feasible } \\
\text { roughness }\end{array}$ \\
\hline$X_{1}$ & rough milling & $13-11$ & $10-14$ & $6.3-25$ & $3.2-25$ \\
$X_{2}$ & $\begin{array}{l}\text { rough milling+ finish milling } \\
\text { rough milling + fine milling + } \\
\text { finish milling }\end{array}$ & $11-8$ & $7-12$ & $6.3-12.5$ & $12.5-3.2$ \\
$X_{3}$ & $\begin{array}{l}\text { rough milling + fine milling + } \\
\text { finish milling+ grinding etc. }\end{array}$ & & $7-6$ & $0.8-3.2$ & $0.4-6.3$ \\
& & $7-6$ & $5-7$ & & \\
$X_{4}$ & & & & & \\
\hline
\end{tabular}

Table $2 Z$ processing scheme

\begin{tabular}{|c|c|c|c|c|c|}
\hline code & $\begin{array}{l}\text { processing } \\
\text { method }\end{array}$ & $\begin{array}{l}\text { economic } \\
\text { size } \\
\text { tolerance }\end{array}$ & $\begin{array}{l}\text { feasible size } \\
\text { tolerance }\end{array}$ & $\begin{array}{l}\text { economic } \\
\text { roughness }\end{array}$ & $\begin{array}{l}\text { feasible } \\
\text { roughness }\end{array}$ \\
\hline$Z_{1}$ & drilling $(\mathrm{D} \leq 15 \mathrm{~mm})$ & $13-11$ & $10-14$ & $12.5-50$ & $6.3-50$ \\
\hline$Z_{2}$ & drilling(D>15mm) & $12-10$ & $9-12$ & $12.5-50$ & $6.3-50$ \\
\hline$Z_{3}$ & drilling- rough bearizing & $13-12$ & $11-14$ & $12.5-25$ & $6.3-25$ \\
\hline$Z_{4}$ & $\begin{array}{l}\text { drilling- rough bearizing -finish } \\
\text { bearizing }\end{array}$ & $11-9$ & $8-12$ & $3.2-6.3$ & $1.6-12.5$ \\
\hline$Z_{5}$ & $\begin{array}{l}\text { drilling- rough bearizing - finish } \\
\text { bearizing - semi finished hinge }\end{array}$ & $9-8$ & $7-10$ & $3.2-6.3$ & $1.6-12.5$ \\
\hline$Z_{6}$ & $\begin{array}{l}\text { drilling- bearizing - semi finish } \\
\text { bearizing - finish ream }\end{array}$ & $7-6$ & $5-8$ & $0.8-3.2$ & $0.4-6.3$ \\
\hline$Z_{7}$ & drilling - semi-fine boring & $11-10$ & $9-12$ & $1.6-6.3$ & $0.8-12.5$ \\
\hline$Z_{8}$ & $\begin{array}{l}\text { drilling- semi-fine boring - } \\
\text { fine boring }\end{array}$ & $9-7$ & $6-10$ & $0.8-3.2$ & $0.4-6.3$ \\
\hline$Z_{9}$ & $\begin{array}{l}\text { drilling- rough bearizing - finish } \\
\text { bearizing - fine boring }\end{array}$ & $7-5$ & $5-8$ & $0.2-0.4$ & $0.1-0.8$ \\
\hline
\end{tabular}




\section{The extension associated reasoning of characteristics machining operation}

The extension comprehensive correlation calculation. Aiming at the recognition feature for process design, the association relationship between material, size tolerance, roughness and machining method is built after the shape classification of characteristics. Due to the value difference between each index is larger; the similarity measure has certain difficulty, each indicator can be converted to the associated degree value by adopting the method of extension dependent function in order to facilitate comparative analysis. The calculations of extension correlation function are as follows [6]:

(1)Calculation of distance: set $\mathrm{x}$ as any point on the real axis, $X_{0}=\langle a, b\rangle$ is any interval on the real field, then the distance is

$$
\rho\left(x, X_{0}\right)=\left|x-\frac{a+b}{2}\right|-(\mathrm{b}-\mathrm{a}) / 2
$$

And here, $\langle a, b\rangle$ is the open interval, also it can be used for the closed interval, as well as for half-closed interval.

(2) The calculation of place value: suppose the equal interval and acceptable interval of index is $X_{0}=\langle a, b\rangle 、 X=\langle c, d\rangle$ respectively, and $X_{0} \in X$, the distance value composed by interval $X_{0}$ and $X_{0}$ of the point $x$ is defined as follows:

$$
D\left(x, X_{0}, X\right)=\left\{\begin{array}{cc}
\rho(x, X)-\rho\left(x, X_{0}\right) & \rho(x, X) \neq \rho\left(x, X_{0}\right) \text { and } x \text { do not belong to } X_{0} \\
\rho(x, X)-\rho\left(x, X_{0}\right)+a-b & \rho(x, X) \neq \rho\left(x, X_{0}\right) \text { and } x \text { belong to } X_{0} \\
a-b & \rho(x, X)=\rho\left(x, X_{0}\right)
\end{array}\right.
$$

(3) The calculation of correlation degree: Correlation function of point $\mathrm{x}$ about the interval $X_{0}$ and $X$ is as follows:

$$
k(x)=\left\{\begin{array}{cc}
\rho\left(x, X_{0}\right) / D\left(x, X_{0}, X\right)-1 & \rho(x, X)=\rho\left(x, X_{0}\right) \text { and } x \text { do not belong to } X_{0} \\
\rho\left(x, X_{0}\right) / D\left(x, X_{0}, X\right) & \text { the others }
\end{array}\right.
$$

(4)the calculation of comprehensive correlative degree: Assume that the weight coefficient of each index factor is $\lambda_{j}$, and meet $\sum_{j=1}^{d} \lambda_{j}=1$, the comprehensive correlation degree for instance objects is

$$
K_{i}(F E)=\sum_{j=1}^{d} \lambda_{j} k\left(c_{i j}(F E)\right)=\sum_{j=1}^{d} \lambda_{j} k\left(\mathrm{v}_{i j}\right)
$$

The processing method correlation of identify characteristics.Because the processing parts is composed of various processing characteristics, suppose the sequence of processing characteristics is , the recognition of the machining features have the blank materials, shape, size tolerance and surface roughness value, therefore, the association mapping model is built as fig. 2 . 


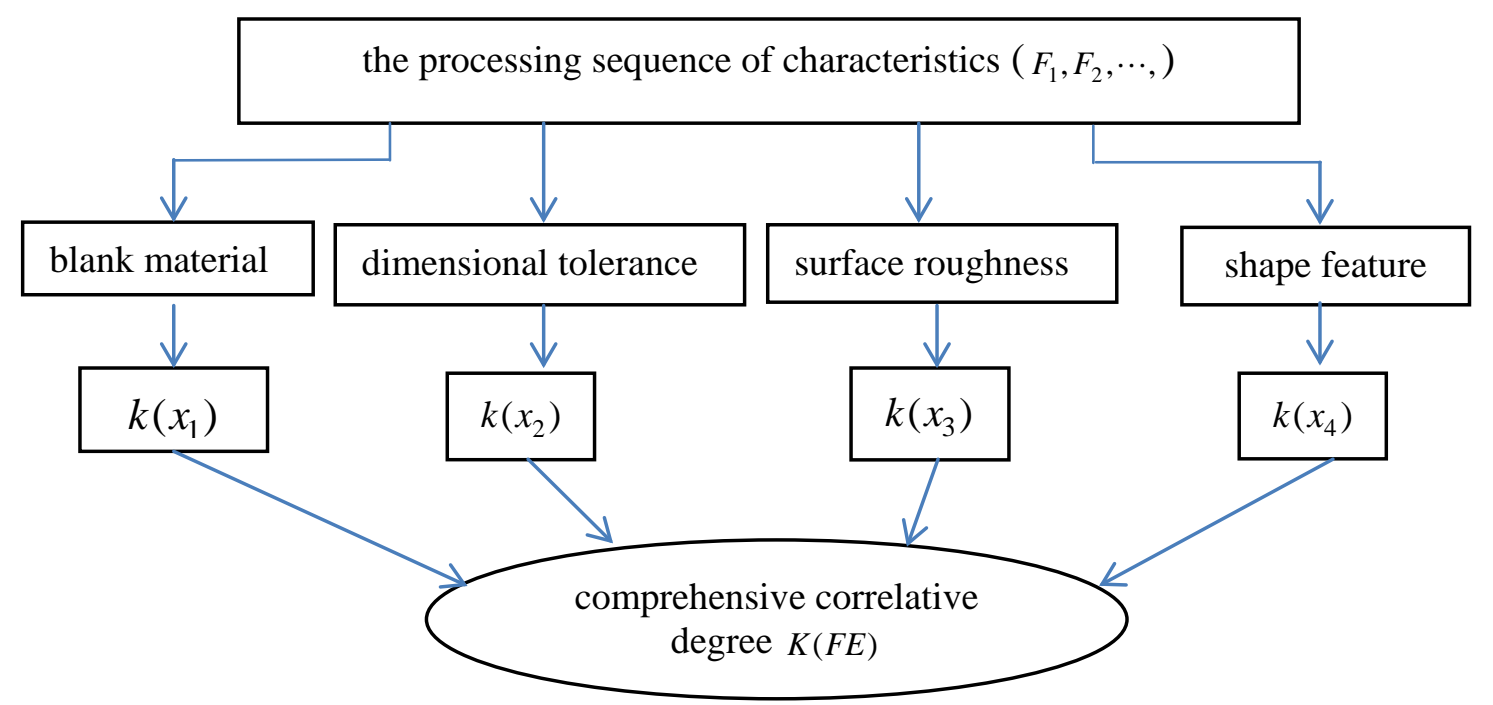

Fig.2 comprehensive correlation mapping of machining feature

\section{Application examples}

As shown in figure 3, the blank of parts is envelope; its upper surface has a $1 \mathrm{~mm}$ allowance offset. For brevity, suppose the materials are 45 \# steel, form and position tolerance was thought to be guaranteed mainly by the clamping and machine tools. The features after automatic recognition are as shown in table 3 .

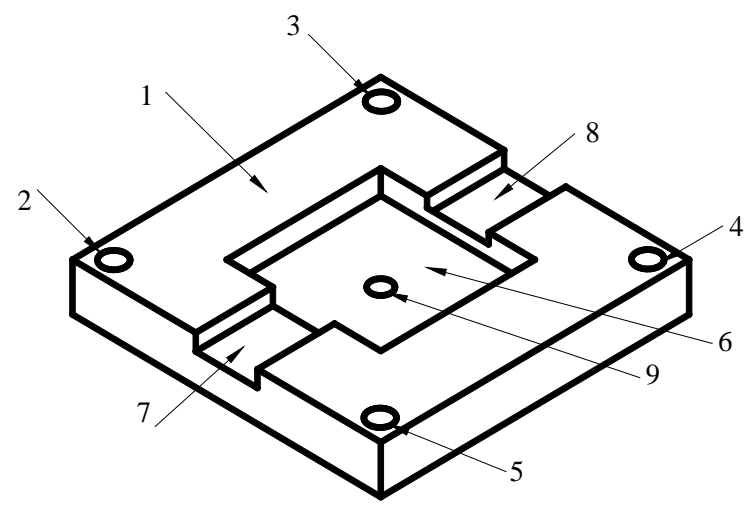

Fig.3 3 D part drawing

Table 3 the recognition characteristics

\begin{tabular}{clccc}
\hline $\begin{array}{c}\text { The feature } \\
\text { number }\end{array}$ & Features & $\begin{array}{l}\text { Dimensional } \\
\text { tolerance }\end{array}$ & Roughness & Others \\
\hline 1 & surface & IT 9 & 1.6 & \\
$2,3,4,5$ & hole & IT 11 & 3.2 & $18 \mathrm{~mm}$ \\
6 & plane cavity & IT 7 & 0.8 & \\
7 & plane cavity & IT 7 & 0.8 & \\
8 & plane cavity & IT 7 & 0.8 & \\
9 & hole & IT 12 & 12.5 & $8 \mathrm{~mm}$ \\
\hline
\end{tabular}

Features precision value is mainly evaluated according to the highest precision grade, the

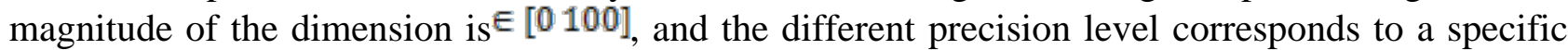
percentile, which is as shown in table 4.

Table 4 index interval value of precision grade evaluation

\begin{tabular}{lrcccccccccc}
\hline roughness (Ra) & $>25$ & 25 & 12.5 & 6.3 & 3.2 & 1.6 & 0.8 & 0.4 & 0.2 & 0.1 & $<0.1$ \\
\hline $\begin{array}{l}\text { dimensional } \\
\text { tolerance (IT) }\end{array}$ & $>13$ & 13 & 12 & 11 & 10 & 9 & 8 & 7 & 6 & 5 & $<5$ \\
\hline percentile & 0 & 10 & 20 & 30 & 40 & 50 & 60 & 70 & 80 & 90 & 100 \\
\hline
\end{tabular}


From this, dimensional tolerance and roughness values of table 1 and table 2 are converted to the equal interval and acceptable interval of the extension relationship by using 4 . The result of calculation is that the most close to the processing method of characteristics 1 is $X_{2}$, the most close to the processing method of characteristics $2 、 3 、 4 、 5$ is $Z_{4}$, the most close to the processing method of plane cavity characteristics $6 、 7 、 8$ is $X_{3}$, the most close to the processing method of number 9 holes is $Z_{1}$.

\section{Conclusions}

The processing technology based on the characteristics can use the feature parameters of the product model and combine the parts blank defined and the processing method of typical features, establish the relationship between the machining method from blank parts, dimensional tolerance, surface roughness and shape feature, etc. In this paper, the manufacturing feature is recognized by the existing 3D software, and the processing rules of features are defined, the typical machining method is given for the non-hole class characteristics and hole class characteristics, size tolerance achieved and surface roughness are given. The association relationship between processing technology and processing method is built based on extension comprehensive correlation functions, the typical processing method which is most closely linked to recognition feature is choose as the processing method of this method. The application instance shows the better operability of this method, and can adapt to the current characteristics of the typical CAM software in the automatic process selection problem.

\section{Acknowledgements}

Thanks to the funding of Zhejiang Province Natural Science Fund Project (LY13E050021) and Jiaxing Science and Technology Plan Projects (2014BY28004).

\section{References}

[1]Shen Bin, Ouyang Huabing. Feature processing technology and its implementation in CAMWorks[J]. Machine Tool \& Hydraulics, Vol. 39 (2011) No. 4, p.33-36.

[2] Yun Zhidong, Li Haibiao. The Application of FBM Technology in Automatic NC Programming[J]. Modular Machine Tool \& Automatic Manufacturing Technique, 2011, (1): p.90-93.

[3] Dae-Hyuk Chung, Suk-Hwan Suh. ISO 14649-based nonlinear process planning implementation for complex machining. Computer-Aided Design, 2008, 40, p.521-536.

[4] Matthieu Rauch, Raphael Laguionie, Jean-Yves Hascoet, et al. An advanced STEP-NC controller for intelligent machining process. Robotics and Computer-Integrated Manufacturing, 2012, 28, p.375-384.

[5] Huang Rui, Zhang Shusheng, Bai Xiaoliang. Manufacturability Driven Interacting Machining Feature Recognition Algorithms for 3D CAD Models [J]. Journal of Computer-Aided Design \& Computer Graphics, Vol. 25 (2013) No. 7,p. 1089-1098.

[6] Li Qiao-xing, Liu Si-feng. A Method to Construct the General Location Value and General Elementary Dependent Function[J]. Systems Engineering, Vol. 24 (2006) No. 6,p. 116-118. 